\title{
Midwife conceptualizations of clinical leadership in the labor ward of district hospitals in KwaZulu-Natal, South Africa
}

This article was published in the following Dove Press journal: Journal of Healthcare Leadership

\section{Solange Mianda \\ Anna S Voce}

Discipline of Public Health Medicine, School of Nursing and Public Health, College of Health Sciences, University of KwaZulu-Natal, Durban, South Africa
Correspondence: Solange Mianda Discipline of Public Health Medicine, School of Nursing and Public Health, College of Health Sciences, University of KwaZulu-Natal, Room 236, 2nd Floor George Campbell Building, Howard College Campus, 238 Mazisi Kunene Road, Glenwood, 404I, Durban, South Africa

Tel +278427943I0

Email solange.zoe60@gmail.com
Background: In South Africa, inadequately skilled health care providers and poor clinical leadership are continually linked to preventable perinatal and maternal mortality, which calls for improved clinical skills among health care providers and for strong clinical leadership at the bedside. Very little has been done to ensure clinical leadership at the bedside in the labor ward of district hospitals. One strategy implemented has been the appointment of District Clinical Specialist Teams, introduced to improve the quality of maternal and child health care in district hospitals and clinics through the provision of clinical leadership as an outreach activity. However, the strengthening of clinical leadership at the bedside remains neglected. Further, clinical leadership in the literature is not conceptualized in the same way across settings.

Aim: To explore midwife conceptualizations of clinical leadership in the labor ward of district hospitals in KwaZulu-Natal.

Methods: Iterative data collection and analysis, following the Corbin and Strauss grounded theory approach, was implemented. In-depth interviews were carried out with the midwifery members of the District Clinical Specialist Teams in KwaZulu-Natal. The emergent theoretical framing of clinical leadership was presented and discussed at a workshop with broader midwifery representation, leading to a final proposition of the conceptualization of clinical leadership among midwives.

Results: The emergent conceptualization of clinical leadership comprised five major dimensions: the definition of clinical leadership, the context in which clinical leadership takes place, the conditions related to clinical leadership, the actions and interactions involved in clinical leadership, and the effects of clinical leadership.

Conclusion: Clinical leadership is an emergent phenomenon arising from dynamic interactions in the labor ward and the broader health system, which converge to attain optimal patient care. Clinical leadership is not being understood from a traditional hierarchical perspective, as vested only in a positional leader.

Keywords: bedside care, clinical leadership, frontline health workers, midwives, grounded theory, leadership, qualitative research, patient outcomes

\section{Introduction and background}

Globally, since 2011, preventable perinatal mortality has remained unchanged, suggesting poor care during pregnancy and childbirth. ${ }^{1}$ To address the burden of preventable perinatal mortality, the Lancet Group called for strong leadership at both country and institutional levels. ${ }^{1}$ In South African district hospitals, where a majority of births occur, ${ }^{2}$ preventable perinatal and maternal deaths have been linked to inadequately skilled health care providers and poor clinical leadership. ${ }^{3,4}$ 
Lack of fetal monitoring and not detecting fetal distress, not responding to abnormalities, and delays in referral have been identified as the most common preventable factors contributing to mortality., ${ }^{3,4}$ These findings have led to the call to improve clinical skills among health care providers and to strengthen clinical leadership at the bedside. ${ }^{3,4}$ Several initiatives have been implemented to improve the clinical skills of health care providers, for example, through expanding training in the Essential Steps in the Management of Obstetric Emergencies. ${ }^{5,6}$ Very little has been done to ensure clinical leadership at the bedside in the labor ward of district hospitals.

One strategy implemented in South Africa to address clinical leadership has been the appointment of District Clinical Specialist Teams (DCSTs). ${ }^{7,8}$ DCSTs were introduced by the National Department of Health as part of the strategy to improve the quality of maternal and child care, through the strengthening of clinical leadership and clinical governance, as an outreach activity to district hospitals. ${ }^{7,8}$ However, the clinical leadership required within district hospitals is not fully described. Furthermore, while the Nursing Strategy for South Africa recommends that nursing leadership should be developed, and stipulates that it is the joint responsibility of the National and Provincial Departments of Health and the South African Nursing Council to do so, it is silent on clinical leadership at the bedside. ${ }^{9}$

In the labor wards of district hospitals, the majority of births are midwife-led, with doctors on call to deal with complications and emergencies. ${ }^{2}$ Registered midwives have the ability to influence the care practices in the ward through clinical leadership. ${ }^{10}$ Midwives are called to use their knowledge, skills, and expertise to care effectively for women and babies, but often they are neither clear about nor have been prepared for the clinical leadership role. ${ }^{10,11}$ For registered midwives to provide quality care, it becomes imperative that they understand the clinical leadership role. ${ }^{10}$ It is argued that clinical leadership unpreparedness in midwifery and nursing is due to the lack of an agreed definition. ${ }^{12}$ There is no standard definition for clinical leadership provided in the literature. ${ }^{13}$ A review of conceptualizations of clinical leadership from the perspective of frontline health care providers shows that clinical leadership is conceptualized differently across health settings. ${ }^{14}$

In view of the diverse conceptualizations of clinical leadership identified in the literature, it was found necessary to explore how midwives conceptualize clinical leadership in the labor ward of district hospitals labor in KwaZulu-Natal (KZN), South Africa.

\section{Aim}

The aim of this study was to explore midwife conceptualizations of clinical leadership in the labor ward of district hospitals in KZN, South Africa.

\section{Methods \\ Design}

A grounded theory methodology according to Corbin and Strauss ${ }^{15}$ was utilized to explore midwife conceptualizations of clinical leadership. This methodology seeks to generate a practical theory for a phenomenon where there is little available evidence, ${ }^{15,16}$ and was considered most appropriate in a context where clinical leadership is not well defined.

\section{Respondents}

Purposive heterogeneous sampling ${ }^{17}$ was implemented, and the following types of key informants were selected for the study: advanced diploma midwifery members of the DCSTs in KZN; advanced diploma midwives in leadership positions in labor wards of district hospitals; academicians responsible for midwifery training in KZN; and district and provincial level representatives from the Directorate for Maternal, Child and Women's Health in the Provincial Department of Health. Respondents were recruited through email and telephonic invitation.

Ethical approval to conduct the study was provided by the University of KwaZulu-Natal Biomedical Research Ethics Committee (ref. BE389/14). Permission to conduct the study was obtained from the KwaZulu-Natal Provincial Department of Health (ref. HRKM326/14). Prior to commencement of the in-depth interviews, information sheets containing the aim, objectives, and processes of the study, accompanied by a consent form, were emailed to key informants. Those who agreed to take part in the study returned a copy of the signed consent form. Participation in the study was voluntary, with the option to withdraw at any stage without any penalty.

\section{Procedure}

The study was conducted in two phases, with Phase I comprising in-depth interviews and Phase II comprising a feedback and consensus building workshop.

\section{Phase I: in-depth interviews}

The researcher (SM) conducted individual in-depth face-toface and telephonic audio-recorded interviews with eight advanced midwifery members of the DCSTs, between April 2015 and January 2016, in a location of each respondent's 
choice. An interview guide using open-ended questions guided the in-depth interview, ${ }^{18}$ covering the following topics: understandings of clinical leadership, purpose of clinical leadership, people responsible for providing clinical leadership in the labor ward, roles and responsibilities of clinical leaders, competencies for effective clinical leadership, and how clinical leadership should be provided in the labor ward. Interviews were conducted in English and lasted between 45 minutes and 1 hour.

In keeping with the Corbin and Strauss approach to grounded theory, data collection and analysis occurred iteratively. ${ }^{16}$ After each interview, the transcript was analyzed in three stages. In the first stage of open coding, the researcher read and re-read the transcript, assigning labels to chunks of data. In the second stage of axial coding, codes with similar meaning were grouped together, as were codes that were related to each other. The final stage of selective coding led to the identification of major themes. In conjunction with the coding process, memos were written about the researchers' impression of the data. Phase I resulted in an initial theoretical proposition pertaining to conceptualizations of clinical leadership, the context in which clinical leadership takes place, the conditions related to clinical leadership, the actions and interactions involved in clinical leadership, and the effects of clinical leadership.

\section{Phase II: feedback consensus building workshop}

A workshop was facilitated (SM and AV) to provide feedback on Phase I of the study and to generate additional insights. Workshop participants comprised all the interviewed advanced midwifery members of the DCSTs (eight), advanced diploma midwives in leadership positions in the labor wards of district hospitals (five), academicians responsible for midwifery training in KZN (two), and district (one) and provincial (one) level representatives from the Directorate for Maternal, Child and Women's Health in the Provincial Department of Health. The workshop was video-taped; the researcher (SM) listened to the recording many times to pick up consensus and disagreements with the conceptualizations of clinical leadership that arose in Phase I, as well as to discern different emphases provided by the workshop participants. SM analyzed the audio recording of the workshop with a focus on attaining meaning saturation for the categories identified in Phase I. Additionally, the workshop served the purpose of ensuring trustworthiness of the research findings by introducing member check as a technique for enhancing credibility. ${ }^{19}$

\section{Findings}

Midwife conceptualizations of clinical leadership in labor wards in KZN emerged organized in five major themes. The themes include the following: definition, the context in which clinical leadership takes place, the conditions related to clinical leadership, the actions and interactions involved in clinical leadership, and the effects of clinical leadership. While presented as separate, the themes are linked, with much overlap between them.

\section{The definition (conceptualization of clinical leadership)}

Clinical leadership was defined in terms of what it is meant to achieve: improved patient outcomes, optimal patient care, teamwork, and professional development.

Clinical leadership is about improving the quality of patient care. So, all in all, it will be to improve service delivery. [Respondent 6]

With clinical leadership in the labour ward, we are aiming at reducing mortality and morbidity and improving health and saving mothers and babies. [Respondent 1]

With clinical leadership, you monitor poor progress of labor, and other staff can learn as you [clinical leader] are asking questions. You are working with them [other staff], and you look at the management together with the people that are there [other staff]. You work together and learn together. [Respondent 5]

\section{The context in which clinical leadership takes place}

Two key points emerged concerning where clinical leadership is provided: firstly, that clinical leadership is provided within the labor ward and secondly, at the patient bedside.

Clinical leadership is not taking yourself away in the duty room and you lead from there. It means being hands on. If you are doing most of the work with patients at the bedside that's an opportunity to teach and provide clinical leadership. [Workshop]

\section{Conditions related to clinical leadership}

Fundamental competencies were identified, which everyone must have for clinical leadership to be effective. However, it was felt that positional clinical leaders must exhibit an advanced level of competency. The list of required competencies for effective clinical leadership identified by respondents is presented in Table 1 in no particular order. 
Table I Competencies required for effective clinical leadership

\begin{tabular}{|l|l|}
\hline Domain & Clinical leadership competencies \\
\hline Personal & Resilience \\
& Flexibility \\
& Confidence \\
& Positive attitude \\
& Motivation \\
& Self-directed learning \\
\hline Relational & Effective communication \\
& Role modeling \\
& Approachability \\
& Visibility \\
& Team work \\
\hline Clinical & Clinical knowledgeability and expertise \\
& Accountability for evidence-based practice \\
& Clinical problem solving \\
& Clinical decision making \\
\hline Organizational & Delegation \\
& Mentoring \\
& Supervision \\
& Teaching \\
& Standard setting \\
& Team management \\
& Resource management \\
& Performance management \\
& Management of the environment of care \\
\hline
\end{tabular}

Differentiating clinical leaders from other health care leaders, is their particular clinical expertise, and the appropriate application of these generic leadership competencies to specific clinical settings.

She must have certain clinical expertise. She must know these procedures must be done this way. [Respondent 2]

She must have clinical skills so that she can guide the subordinate. [Respondent 4]

The main thing is clinical competence [...]. Clinical leadership has to do with knowledge and mostly skills, especially in terms of clinical management. [Respondent 8]

Since clinical expertise is fundamental to effective clinical leadership, self-directed learning was reported as an essential competency for clinical leaders and the maintenance and advancement of clinical competencies.

\section{Actions and interactions related to clinical leadership}

The responsibilities of clinical leadership were seen as being shared and performed by all health care providers in the labor ward and best performed by those who exhibit clinical leadership competencies. While clinical leadership may be shared, the need was identified for appointed positional clinical leaders. The operational manager was identified as the appointed positional clinical leader, overall responsible for the provision of clinical leadership in the labor ward, for overseeing the functioning of the unit, and of the staff, and for ensuring optimal bedside patient care.

The labour ward is run by different people, but it is the operational manager who is leading the team. [Respondent 2]

The operational managers are stationed in the labour ward; they are [the] people who are hands on, in patient care. [Respondent 1]

The operational manager is always there to work, to guide, to support the other staff, to train if there is a need. That is why she needs to be the most knowledgeable person. [Respondent 8]

Operational managers as positional leaders are also responsible for the organization of the care processes, ensuring better performance in the labor ward, patient and staff wellbeing, and clinical governance tasks.

Operational managers should review the performance of the labor ward. They conduct clinical audit and perinatal mortality meetings. When they know the causes of mortality, they can teach their staff to avoid making the same mistakes in future. [Respondent 4]

As a role that can be shared, all health care providers providing patient care were identified as responsible for providing clinical leadership.

Clinical leadership is a responsibility for all midwives.

[Workshop]

Clinical leadership is not only for midwifery, it is also

for doctors. [Respondent 3]

All health care providers in the labor ward were regarded as contributing to optimal bedside patient care, enabling professional development, and creating an enabling environment for optimal bedside patient care.

\section{Enabling optimal bedside patient care}

An important effect of clinical leadership is the provision of optimal bedside care. Toward enabling optimal bedside care, positional clinical leaders were required to model and ensure adherence to protocols of management and evidence-based practice for the management of normal labor, complications, and emergencies. Positional clinical leaders ensure the effective implementation of processes of patient care, from initial assessment to ongoing management.

Reportedly, in monitoring and auditing adherence to the protocols of management, positional clinical leaders influence the quality and standards of care in the labor ward. Ward rounds were declared to be opportunities for positional 
clinical leaders to monitor and update patient care plans. Further, positional clinical leaders were called to identify best practices within other units and institutions and to create platforms where their own teams can benchmark and share information with other professionals.

During ward round, first of all is the review of patients' chart $[\ldots]$ to pick up anything that has gone wrong with the patient $[\ldots]$ you must be able to come up with a clear diagnosis supported by documentation. Then the plan of management should be clear and documented for everybody to follow. And after that allow people to ask questions so that at the end of the ward round everybody is clear on the management of that patient and what will be the clinical consequences of bad management. [Respondent 5]

\section{Enabling professional development for optimal bedside care}

As facilitators of professional development at the bedside, clinical leaders were called on to provide staff with the information, support, and resources to develop clinical problemsolving and decision-making skills. At the bedside, clinical leaders are able to identify skill deficiencies among staff and drawing on their own clinical expertise, have the opportunity to equip them with required skills. Positional clinical leaders can strategically create teaching opportunities at the bedside using teaching approaches that best fit their context to develop skills in staff.

Some teachings should not be planned. If you have to plan, time will not be there because of staff shortage and meetings. But create the opportunity maybe with one patient. You say: Let us learn from that one patient. [Workshop]

As clinical mentors, clinical leaders orient and induct new staff, introducing them to patient care protocols and guidelines. Clinical leaders must use skill assessment, mentorship agreements, and mixed approaches to develop skills in health care providers. Drawing from their leadership skills, positional clinical leaders can delegate leadership tasks to other health care providers. Midwives viewed delegation as a means to build leadership skills in staff.

[...]. It doesn't have to be you all the time, but you can delegate, allocate some tasks to the staff in your department [...]. And from time to time, because you have additional expertise, you can intervene here and there, so that you can guide the staff and build leadership within the younger ones in your department. [Respondent 7]

\section{Creating an enabling environment for optimal bedside care}

Positional clinical leaders must create an enabling environment for optimal bedside care by making sure that the environment of care is inviting and safe. Positional clinical leaders must ensure the availability of staff, resources, and equipment in the labor ward to provide optimal bedside patient care.

You need to have equipment that is functional and checked on a daily basis [...]. We do not need a lot of equipment, but the basics, so that we can identify the risks among the mothers and act accordingly. [Respondent 7]

Clinical leaders also must ensure the availability of protocols for the management of normal labor, complications, and emergencies.

\section{You need to bring in your policies and protocols for the management of obstetric emergencies [...]. You need to know what guidelines to use while providing normal labor, high risk pregnancies or post-natal care. [Respondent 1]}

Clinical leaders create an enabling environment for optimal bedside care by creating a good climate, where relationships can be built and maintained among health care providers within a multidisciplinary team. Clinical leaders are described as team players and team builders.

Clinical leaders must be team builders, they mustn't separate doctors from midwives/nurses. They bring the team together, into one unit. Whatever they are doing, they plan with doctors, therapists and other professionals. [Respondent 1]

A clinical leader must be able to work with midwives, doctors and deal with lower categories [sic]. The clinical leader must know how to make the team function so that quality care is provided. [Respondent 2]

\section{Effects of clinical leadership at the bedside}

Clinical leadership was reported to result in positive outcomes in patients, among health care providers, and in the environment of care. Firstly, clinical leadership may result in better patient experiences and outcomes.

Clinical leadership is about improving the quality of patient care $[. .$.$] . So, all in all, it will improve service delivery.$ [Respondent 6]

Secondly, clinical leadership could result in professional development, staff motivation, and job satisfaction. 
When healthcare providers have been well equipped, there will be job satisfaction and that will improve productivity within your staff. And if they see that there is somebody who is caring more about them, this will definitely yield better outcomes. [Respondent 7]

Thirdly, clinical leadership may result in an improved environment of care, promoting a good climate for inter- and intra-professional collaborations for health care providers, comprising improved communication, teamwork, functional teams, and continuity of care at the bedside.

\section{Discussion}

There is no consistent conceptualization of clinical leadership in the literature. Predominantly, clinical leadership, particularly of frontline health care providers, has been conceptualized in high-income country (HIC) health settings. ${ }^{14}$ Clinical leadership must be defined based on how it is experienced in local clinical settings. ${ }^{13}$ Therefore, the purpose of this study was to describe clinical leadership as conceptualized by midwives in the labor ward of district hospitals in KZN, South Africa. South Africa is categorized as an upper middleincome country, ${ }^{20}$ but is characterized by health inequalities, ${ }^{21}$ affecting resourcing, quality of care, and health outcomes. ${ }^{21-23}$ Further challenges in South African health systems include the shortage of health care providers, clinical experts generally, and midwives in particular. ${ }^{24}$ Most district hospital labor wards, including those in KZN, are staffed with newly qualified health care providers who are inexperienced and in need of clinical mentorship themselves. ${ }^{25}$

In this study conducted in KZN, midwives conceptualized clinical leadership as an emergent phenomenon arising from dynamic interactions within the labor ward and the broader health system, which converge to produce optimal patient care. Clinical leadership for optimal patient care was conceptualized as having a focus at the bedside, as a set of competencies, and as a set of responsibilities, albeit shared, performed by positional clinical leaders and all health care professionals working in the labor ward. ${ }^{14}$ Furthermore, midwives described clinical leadership in terms of intermediary dynamics required for optimal patient care, that is, professional development, teamwork, and an enabling environment. The conceptualization of clinical leadership by midwives in this study is consistent with the literature from HIC. ${ }^{14}$

The emphasis on clinical leadership being performed in the labor ward, at the bedside, is consistent with the emphasis found in other studies. ${ }^{14,26-30}$ The bedside presents an excellent platform for ensuring optimal patient care and assessing the dynamics that influence processes of care. Skill deficiencies among staff may be identified, knowledge of and adherence to standard protocols of management may be monitored, and the adequacy of the environment of care can be assessed. ${ }^{31}$ Initiatives implemented in South Africa to improve the clinical skills of health care providers and their adherence to standard protocols of management, and the environment of care, for example, through the simulation training in the Essential Steps in the Management of Obstetric Emergencies, focus on bedside management. ${ }^{5,6}$

In the description of the set of competencies required by clinical leaders, there was an emphasis on the need for clinical expertise, in addition to other critical leadership competencies. ${ }^{29,32,33}$ It is the clinical expertise of clinical leaders that critically distinguishes clinical leaders from health service managers. ${ }^{14}$ Clinical leaders exercise clinical leadership in the labor ward, drawing on expert rather than on positional power. ${ }^{34,35}$ However, where positional clinical leaders are not as clinically competent as their subordinates, other leadership competencies, such as humility and delegation, are required to allow other frontline health care workers to exercise their leadership role by virtue of their clinical expertise. ${ }^{35}$

Clinical leadership, conceptualized as a shared responsibility performed by every frontline health care provider, diverges from traditional hierarchical autocratic models of leadership, particularly present in the labor ward where the doctor by default is seen as the clinical leader and as having more authority and control. ${ }^{36,37}$ This is at odds in settings like the labor ward of district hospitals in KZN, where clinical care is mostly provided by midwives.$^{38}$ In settings where the doctor is regarded as the clinical leader and is not always present in the labor ward, in case of complications and emergencies, role confusion and blurred boundaries of responsibility may result in suboptimal care. ${ }^{39-41}$

Nevertheless, conceptualizations of clinical leadership as a shared role support shared values, joint responsibility, mutual trust, and teamwork; are empowering of midwifeled care; and are premised on everyone knowing their specific role in the management of clinical emergencies, according to the agreed-upon evidence-based protocols of management. ${ }^{36,37,42-44}$ Shared clinical leadership is a type of situational approach to leadership, dependent on positive human relations and influence. ${ }^{37,45-47}$

A bidirectional influence may exist between clinical leadership and teamwork, with effective clinical leadership contributing to teamwork and teamwork in turn contributing to clinical leadership. As a set of responsibilities related specifically to ensuring teamwork, clinical leadership must 
contribute to a good climate, where relationships among health care providers within a multidisciplinary team can be built and maintained. One major cause for high mortality in labor wards is the lack of teamwork among multiple health care providers at the bedside. ${ }^{48}$ Hence, clinical leaders must facilitate effective team processes through coordination, communication, cohesion, conflict management, decision-making, social relationships, mutual respect, and shared roles. ${ }^{42,49}$ However, in a context of traditional hierarchical autocratic models of leadership, teamwork might be thwarted. ${ }^{41,50}$

As a set of responsibilities for ensuring an enabling environment for optimal bedside patient care, positional clinical leaders are tasked to ensure the environment of care is inviting and safe for patient and health care providers. Positional clinical leaders ensure there are sufficient staff, resources, and equipment to provide optimal bedside care and facilitate professional development. ${ }^{51}$

\section{Strength of the study}

This study is one of the first empirical studies to provide conceptualizations of clinical leadership outside of an HIC setting. The focus in this study was on conceptualizations of clinical leadership by midwives in KZN, South Africa. The study highlights the different conceptual characteristics of clinical leadership, to be implemented at the bedside, in the labor ward, to produce optimal patient care.

\section{Limitations}

The findings of this study should be interpreted in the light of few limitations. This study is limited to midwife conceptualizations of clinical leadership in the labor ward of district hospitals in KZN. The perspectives of other frontline health care providers have not been considered in this study. It would be essential to conduct further research to obtain a multidisciplinary view of clinical leadership and to develop a holistic conceptualization of clinical leadership in this care setting.

\section{Conclusion}

Clinical leadership in resource-constrained labor wards of district hospitals was conceptualized as an emergent phenomenon that is evidenced by optimal patient care, which results from dynamic interactions in the labor ward. The dynamic interactions include clinical leaders with a set of clinical leadership competencies critical to which is clinical expertise, teamwork allowing for shared clinical leadership, and professional development optimized within an enabling environment. Application of these conceptualizations of clinical leadership may contribute to optimal patient care even within the resource $=$ constrained settings of a majority of labor wards in district hospitals in KZN.

A model of intervention for ensuring these conceptualizations of clinical leadership is recommended.

\section{Disclosure}

The authors report no conflicts of interest in this work.

\section{References}

1. Frøen F, Lawn J, Haezell A. Ending preventable stillbirths. An executive summary for The Lancet's series. Lancet; 2016. Available from: http://www.thelancet.com/pb/assets/raw/Lancet/stories/series/ stillbirths2016-exec-summ.pdf. Accessed June 13, 2017.

2. National Department of Health. Guidelines for Maternity Care in South Africa. A Manual for Clinics, Community Health Centres and District Hospitals. Pretoria: South African Department of Health; 2015.

3. Pattinson R, Rhoda N. Saving Babies 2012-2013: Ninth Report on Perinatal Care in South Africa for the PPIP Group. Pretoria: Tshepesa Press; 2014.

4. National Committee for the Confidential Enquiry into Maternal Deaths. Saving Mothers 2011-2013: Sixth Report on the Confidential Enquiry into Maternal Deaths in South Africa. Pretoria: South African Department of Health; 2014.

5. Bateman C. Maternal and infant mortality expert hopeful for South Africa. S Afr Med J. 2009;100(3):141-143.

6. Pattinson R. Improving emergency obstetric care. Int J Gynaecol Obstet. 2010;110(2):87-88.

7. Voce A, Bhana R, Monticelli F. District Clinical Specialist Teams. In: Padarath A, Kling J, English R, editors. South African Health Review 2013/14. Durban; 2013:14. Available from: http://www.hst.org.za/sites/ default/files/DCSTs.pdf. Accessed May 24, 2017.

8. Department of Health, Ministerial Task Team. District Clinical Specialist Teams in South Africa. Pretoria; 2011. Available from: https://www. health-e.org.za/wp-content/uploads/2015/06/Handbook-for-DCSTs. pdf. Accessed July 4, 2017.

9. Department of Health. Nursing Strategy for South Africa, Choose a Healthy Lifestyle. Pretoria; 2008. Available from: http://www.sanc. co.za/archive/archive2008/linked files/nursing-strategy.pdf. Accessed June 13, 2017.

10. Byrom S, Kay L. Midwifery leadership: theory, practice and potential. In: Downe S, Byrom S, Louise S, editors. Essential Midwifery Practice: Leadership, Expertise and Collaborative Working. 1st ed. Chichester: Willey-Blackwell; 2011:248.

11. Fenton K, Phillips N. Developing skills in clinical leadership for ward sisters. Nurs Times. 2013;109(9):12-15.

12. Health Service Executive. National nursing and midwifery clinical leadership development needs analysis HSE executive report. Dublin; 2010. Available from: https://www.choiceforum.org/docs/clinicaldes. pdf. Accessed May 23, 2017.

13. Daly J, Jackson D, Mannix J, Davidson P, Hutchinson M. The importance of clinical leadership in the hospital setting. J Healthc Leadersh. 2014;6:75-83.

14. Mianda S, Voce AS. Conceptualizations of clinical leadership: a review of the literature. J Healthc Leadersh. 2017;9:79-87.

15. Corbin JM, Strauss AL. Basics of Qualitative Research: Techniques and Procedures for Developing Grounded Theory. California: SAGE Publications; 2015.

16. Creswell JW. Qualitative Inquiry and Research Design: Choosing among Five Approaches. 3rd ed. Thousand Oaks: SAGE Publications; 2013.

17. Patton M. 3rd ed. Chap 2: Qualitative Designs and Data Collection. In: Laughton D, Novak V, Journey K, editors, Qualitative Research and Evaluation Methods. London: SAGE publications; 2002. 
18. Edwards R, Holland J, editors. What Is Qualitative Interviewing? 1st ed. London: Bloomsbury Academic; 2013. Available from: http://eprints. ncrm.ac.uk/3276/1/complete_proofs.pdf. Accessed September 19, 2017.

19. Creswell J, Miller DL. Determining validity and reliability in qualitative inquiry. Theory Pract. 2000;39(3):124-131.

20. World Bank. Country Income Groups (World Bank Classification). Country and Lending Groups; 2011. Available from: http://data.worldbank.org/about/country-classifications/country-and-lending-groups. Accessed October 19, 2017.

21. Mayosi BM, Benatar SR. Health and health care in South Africa 20 years after Mandela. N Engl J Med. 2014;371(14):1344-1353.

22. Mayosi BM, Lawn JE, van Niekerk A, et al. Health in South Africa: changes and challenges since 2009. Lancet. 2012;380(9858): 2029-2043.

23. Whiteside A. South Africa's key health challenges. Ann Am Acad Pol Soc Sci. 2014;652(1):166-185.

24. Mabaso MH, Ndaba T, Mkhize-Kwitshana ZL. Overview of maternal, neonatal and child deaths in South Africa: challenges, opportunities, progress and future prospects. Int J MCH AIDS. 2014;2(2):182-189.

25. Rispel LC. Special issue: transforming nursing in South Africa. Glob Health Action. 2015;8:28205.

26. Ennis G, Happell B, Reid-Searl K. Enabling professional development in mental health nursing: the role of clinical leadership. J Psychiatr Ment Health Nurs. 2015;22(8):616-622.

27. Mendis D, Paton C. Perceptions of clinical leadership amongst West Midlands registrars. Int J Leadersh Public Serv. 2014;10(2):108-122.

28. Stanley D, Blanchard D, Hohol A, Hutton M, McDonald A. Health professionals' perceptions of clinical leadership. A pilot study. Cogent Med. 2017;4(1):1-15.

29. Jonas S, McCay L, Keogh B. The importance of clinical leadership. In: Swanwick T, McKimm J, editors. ABC of Clinical Leadership. 1st ed. Oxford: Blackwell Publishing Ltd; 2011:1-3.

30. Wong CA, Cummings GG, Ducharme L. The relationship between nursing leadership and patient outcomes: a systematic review update. J Nurs Manag. 2013;21(5):709-724.

31. Larsson IE, Sahlsten MJ. The staff nurse clinical leader at the bedside: Swedish registered nurses' perceptions. Nurs Res Pract. 2016;2016: $1-8$.

32. Lett M. The concept of clinical leadership. Contemp Nurse. 2002;12(1): $16-21$.

33. Stanley DJ. Clinical leadership and innovation. J Nurs Educ Pract. 2012;2(2).

34. French J, Raven B. The bases of social power. In: Cartwright D, editor. Studies in Social Power. New York: Institute for Social Research; 1959.

35. Lunenburg FC. Power and leadership: an influence process. International J Manag Bus Adm. 2012; 15(1):1-9.

36. Al-Sawai A. Leadership of healthcare professionals: where do we stand? Oman Med J. 2013;28(4):285-287.
37. National Health Services Institute for Innovation and Improvement. Shared leadership underpinning of the MLCF. In: Enhancing Engagement in Medical Leadership. Coventry; 2009. Available from: http:// www.leadershipacademy.nhs.uk/wp-content/uploads/2012/10/776bc9 c27b6e8741d0ff42e593ba44cf.pdf. Accessed May 24, 2017.

38. Giltinane CL. Leadership styles and theories. Nurs Stand. 2013;27(41): 35-39.

39. Chin GS, Warren N, Kornman L, Cameron P. Transferring responsibility and accountability in maternity care: clinicians defining their boundaries of practice in relation to clinical handover. BMJ Open. 2012;2(5):e000734.

40. Holland S, Goodsell R, Keable J. Clinical Guidelines for All Maternity Staff. London: NHS TRUST; 2018.

41. Patton C. Conflict in health care: a literature review. Int J Health Administr. 2014;9(1). Available from: http://ispub.com/IJHCA/9/1/20081. Accessed May 24, 2017.

42. Rankin J, Matthews A, Russell M, Pollard M. Clinical leadership and effective nursing teams. J Nurs Health Care. 2016;4(1).

43. Costanzo T. My "situational leadership style"; 2005. Available from: https://engineering.ucsb.edu/ tacy/capstone/leadership/my_leadership_style.pdf. Accessed September 25, 2017.

44. Mitchell P, Wynia M, Golden R, et al. Core Principles and Values of Effective Team-Based Health Care. Washington, DC; 2012. Available from: https:/www.nationalahec.org/pdfs/vsrt-team-based-care-principles-values.pdf. Accessed September 25, 2017.

45. Doherty J. Strengthening Clinical Leadership in Hospitals: A Review of the International and South African Literature. Johannesburg: Municipal Service Project; 2013. Available from: https://www.wits.ac.za/media/ migration/files/cs-38933-fix/migrated-pdf/pdfs-5/Strengthening clinical leadership in hospitalsVers2.pdf. Accessed May 23, 2017.

46. House RJ, Mitchelle TR. Path-goal to leadership. Adm Sci Q. 1971;16: 321-338.

47. Hilaire F. Leadership theories: Towards a relational model. 2008. Available from: https://pdfs.semanticscholar.org/1e23/69cc50e5995cbd196 d4e30eaae2454fca59a.pdf. Accessed March 20, 2017.

48. Madden E, Sinclair M, Wright M. Teamwork in Obstetric Emergencies. London; 2011. Available from: https://www.rcm.org.uk/learning-andcareer/learning-and-research/ebm-articles/teamwork-in-obstetricemergencies. Accessed July 4, 2017.

49. Mickan S, Rodger S. Characteristics of effective teams: a literature review. Aust Health Rev. 2000;23(3):201-218.

50. Hagbaghery M, Imahvash S, Fazlolah A. The factors facilitating and inhibiting effective clinical decision-making in nursing: a qualitative study. BMC Nurs. 2004;3(2).

51. Hughes RG. Nurse at the "sharp end". In: Hughes RG, editor. Patient Safety and Quality: An Evidence-Based Handbook for Nurses. Rockville; 2008:6-30. Available from: https://archive.ahrq.gov/professionals/ clinicians-providers/resources/nursing/resources/nurseshdbk/nurseshdbk.pdf. Accessed June 13, 2017.
Journal of Healthcare Leadership

\section{Publish your work in this journal}

The Journal of Healthcare Leadership is an international, peer-reviewed, open access journal focusing on leadership for the health profession. The journal is committed to the rapid publication of research focusing on but not limited to: Healthcare policy and law; Theoretical and practical aspects of healthcare delivery; Interactions between healthcare and society and evidence-based practices;

\section{Dovepress}

Interdisciplinary decision-making; Philosophical and ethical issues; Hazard management; Research and opinion for health leadership; Leadership assessment. The manuscript management system is completely online and includes a very quick and fair peer-review system. Visit http://www.dovepress.com/ estimonials.php to read real quotes from published authors. 